\title{
Manual therapy directed at the knee or lumbopelvic region does not influence quadriceps spinal reflex excitability
}

\author{
Terry L Grindstaff, PhD, PT, ATC, \\ Creighton University, Omaha, Nebraska, USA \\ Brian G Pietrosimone, PhD, ATC, \\ University of Toledo, Toledo, Ohio, USA \\ Lindsay D Sauer, PhD, ATC, \\ University of Virginia, Charlottesville, Virginia, USA \\ D. Casey Kerrigan, MD, MS, \\ JKM Technologies LLC, Charlottesville, Virginia, USA \\ James T Patrie, MS, \\ University of Virginia, Charlottesville, Virginia, USA \\ Jay Hertel, PhD, ATC, FNATA, FACSM, and \\ University of Virginia, Charlottesville, Virginia, USA \\ Christopher D Ingersoll, PhD, ATC, FNATA, FACSM \\ Central Michigan University, Mt. Pleasant, Michigan, USA
}

\begin{abstract}
Manual therapies, directed to the knee and lumbopelvic region, have demonstrated the ability to improve neuromuscular quadriceps function in individuals with knee pathology. It remains unknown if manual therapies may alter impaired spinal reflex excitability, thus identifying a potential mechanism in which manual therapy may improve neuromuscular function following knee injury.
\end{abstract}

\begin{abstract}
Aim-To determine the effect of local and distant mobilisation/manipulation interventions on quadriceps spinal reflex excitability.
\end{abstract}

\begin{abstract}
Methods-Seventy-five individuals with a history of knee joint injury and current quadriceps inhibition volunteered for this study. Participants were randomised to one of five intervention groups: lumbopelvic manipulation (grade V), lumbopelvic manipulation positioning (no thrust), grade IV patellar mobilisation, grade I patellar mobilisation, and control (no treatment). Changes in spinal reflex excitability were quantified by assessing the Hoffmann reflex (H-reflex),
\end{abstract}

\footnotetext{
(C) 2014 Elsevier Ltd. All rights reserved.

*Address correspondence to Terry L. Grindstaff, Creighton University, School of Pharmacy \& Health Professions, Physical Therapy Department, 2500 California Plaza, Omaha, NE 68178. GrindstaffTL@ gmail.com.

Publisher's Disclaimer: This is a PDF file of an unedited manuscript that has been accepted for publication. As a service to our customers we are providing this early version of the manuscript. The manuscript will undergo copyediting, typesetting, and review of the resulting proof before it is published in its final citable form. Please note that during the production process errors may be discovered which could affect the content, and all legal disclaimers that apply to the journal pertain.
} 
presynaptic, and postsynaptic excitability. A hierarchical linear-mixed model for repeated measures was performed to compare changes in outcome variables between groups over time (pre, post $0,30,60,90 \mathrm{~min})$.

Results-There were no significant differences in H-reflex, presynaptic, or postsynaptic excitability between groups across time.

Conclusions-Manual therapies directed to the knee or lumbopelvic region did not acutely change quadriceps spinal reflex excitability. Although manual therapies may improve impairments and functional outcomes the underlying mechanism does not appear to be related to changes in spinal reflex excitability.

\section{Keywords}

H-reflex; knee pain; mobilization; manipulation

\section{INTRODUCTION}

Decreased quadriceps strength is a common impairment following knee joint injury (Chmielewski et al., 2004, Ingersoll et al., 2008, McLeod et al., 2012, Pietrosimone et al., 2011a, Snyder-Mackler et al., 1994) and is a risk factor for knee osteoarthritis (Segal et al., 2010). This weakness is partially due to arthrogenic muscle inhibition which is defined as reflexive inhibition of musculature surrounding a joint following joint injury (Hopkins and Ingersoll, 2000, Stokes and Young, 1984). Previous studies have indicated decreased spinal reflex excitability, specifically presynaptic and postsynaptic mechanisms contribute to quadriceps inhibition following experimental knee joint effusion (Hopkins et al., 2001, Palmieri et al., 2004).

Traditional quadriceps strengthening rehabilitation interventions do not specifically address quadriceps arthrogenic muscle inhibition (Chmielewski et al., 2004, Pietrosimone et al., 2011b). Interventions which alter sensory output, such as transcutaneous electrical nerve stimulation (TENS) and cryotherapy increase quadriceps voluntary activation in individuals with knee pathology (Pietrosimone et al., 2009). Increased voluntary activation may result from increased spinal reflex excitability, which has been demonstrated following cryotherapy and TENS using a joint effusion model (Hopkins et al., 2002). Graded knee joint mobilisation can increase pain thresholds (Courtney et al., 2010, Moss et al., 2007) and modulate the flexor withdraw reflex in individuals with knee osteoarthritis (Courtney et al., 2009, Courtney et al., 2010), but mechanism for changes in quadriceps function are less understood.

Manual therapy interventions applied at distant sites, such as the lumbopelvic region, have been shown to decrease knee pain (Iverson et al., 2008) with mixed results regarding changes in quadriceps force output and voluntary activation (Grindstaff et al., 2012, Hillermann et al., 2006, Suter et al., 1999, Suter et al., 2000). Furthermore, the duration of change in quadriceps inhibition may range from seconds to minutes. It is not clear if changes in quadriceps muscle activation following manual therapies are dependent on the intensity of the mobilisation (Dishman et al., 2002a, Dishman et al., 2005) or the site of application 
(Dishman et al., 2002b). The purpose of this study was to determine the effect of local and distant mobilisation/manipulation interventions on presynaptic and postsynaptic inhibition of the quadriceps muscle in individuals with existing quadriceps inhibition. Better understanding potential mechanisms of manual therapies will help guide future development of optimal treatment interventions for joint pathologies.

\section{METHODS}

Seventy-five individuals with a history of knee joint injury and current quadriceps inhibition volunteered for this study (Table 1 and Figure 1). All participants were between 18 and 40 years of age and had a body mass index (BMI) below 30. Participants also had current or a history of knee joint pathology, which resulted in quadriceps inhibition. Specifically, we included participants who had diagnosed knee osteoarthritis (grades I-II), patellofemoral joint pain, or participants who have undergone arthroscopic surgery for anterior cruciate ligament reconstruction, meniscotomy, plica removal, or debridement. Quadriceps inhibition was quantified as voluntary activation below 90\% (Pietrosimone et al., 2011a) and confirmed through burst-superimposition testing of the involved limb (Grindstaff et al., 2009). Values above $95 \%$ are considered normal and $100 \%$ indicates complete voluntary activation (Stackhouse et al., 2001, Stevens et al., 2003). Finally participants had to have a measurable quadriceps H-reflex. Presence of quadriceps inhibition and H-reflex were determined during an initial screening session using previously described methods (Grindstaff et al., 2009, Palmieri et al., 2005). Exclusion criteria included pregnancy and other lumbar spine or lower extremity injury within the past six months. All participants read and signed the informed consent form for the study, which was approved by our Institutional Review Board.

All measurements, interventions, and testing were performed on the ipsilateral side of pain/ dysfunction. In the event of bilateral pathology, the participants were asked to determine which leg was more symptomatic. If the participant was not able to differentiate between legs, then a coin toss determined the test limb.

\section{Instrumentation}

H-reflex, paired reflex depression, and recurrent inhibition measurements were collected using surface electromyography (MP150, BIOPAC Systems Inc., Santa Barbara, CA). Signals were amplified (DA100B, BIOPAC Systems Inc.; Gain = 1000) from disposable, 10 $\mathrm{mm}$ pre-gelled $\mathrm{Ag}-\mathrm{AgCl}$ electrodes (EL503, BIOPAC Systems Inc). The EMG signal was band-pass filtered from 10 to $500 \mathrm{~Hz}$ and sampled at $1024 \mathrm{~Hz}$ with a common-mode rejection ratio of $110 \mathrm{~dB}$. H-reflex measurements were elicited using the BIOPAC stimulator module (STM100A, BIOPAC Systems Inc.) with a 200-volt (maximum) stimulus isolation adapter (STMISOC, BIOPAC Systems Inc.), $2 \mathrm{~mm}$ shielded disc electrode (EL254S, BIOPAC Systems Inc.) and a $7 \mathrm{~cm}$ dispersive pad.

\section{Outcomes Procedures}

Spinal Reflex Excitability-Skin preparation and electrode placement were consistent with previously published methods (Palmieri and Ingersoll, 2005, Palmieri et al., 2005). The 
skin was prepared for EMG electrode application by shaving, debriding with fine sandpaper, and cleansing with isopropyl alcohol. The surface EMG electrodes were on the vastus medialis to capture the maximum peak to peak amplitude of the H-reflex for all measurements. A percutaneous stimulating electrode $(2 \mathrm{~mm} \mathrm{Ag}-\mathrm{AgCl})$ was covered with conductive gel and placed superficial to the femoral nerve, lateral to the femoral artery, in the femoral triangle and secured with adhesive tape. A dispersive electrode $(7 \mathrm{~cm}$ diameter, rubber carbon impregnated) covered in conductive gel was placed over the posterior proximal thigh. Participants were in a supine position with the knee in approximately 15 degrees of flexion and asked to keep their hands at their sides with palms open and their eyes open while looking at the ceiling (Kameyama et al., 1989). When these factors are controlled, the reliability of quadriceps $\mathrm{H}$-reflex amplitude measures are good $\left(\mathrm{ICC}_{3,1}=\right.$ 0.97) (Hopkins and Wagie, 2003).

Recruitment curves were derived for each participant by gradually increasing stimulus intensity and examining peak-to-peak measures of the H-reflex and $\mathrm{M}$ response (maximal compound muscle action potential). Stimuli (1 ms square wave pulse) were delivered by increasing the intensity in $0.2-\mathrm{V}$ increments until the maximum peak-to-peak amplitude for the H-reflex (Hmax) was obtained. A 12-sec rest interval was provided after each stimulus. Once Hmax was determined three measures were obtained. Next stimulus intensity was increased in 1-V increments until a plateau was reached in the peak-to-peak amplitude of the $M$ response (Mmax). M response plateau was verified by applying a single measure with maximum stimulator intensity $(200 \mathrm{~V})$. The average Hmax and Mmax were used for data analysis.

Paired Reflex Depression (PRD)-presynaptic inhibition-Methods to derive PRD measures were consistent with previously established protocol (Trimble et al., 2000). Briefly, a pair of stimuli ( $80 \mathrm{~ms}$ interpulse interval) were delivered to the femoral nerve while the EMG response was measured in the quadriceps muscle. The stimulus intensity was set to elicit H-reflexes at $10 \%$ of Mmax amplitude. The depression of the second H-reflex relative to the first (\% depression) was used for data analysis. Eight measures were obtained, recorded, and averaged to improve the intrasession reliability (Earles et al., 2002).

Recurrent Inhibition-postsynaptic inhibition-Recurrent inhibition was collected by first eliciting a conditioning stimulus to the tibial nerve with an electrode in the popliteal fossa and followed by a stimulus to the femoral nerve at the femoral triangle. The conditioning stimulus, at an intensity to elicit a soleus Mmax was applied to the tibial nerve $22 \mathrm{~ms}$ prior to a second stimulus applied to the quadriceps at $10 \%$ Mmax. The conditioning stimulus was used to stimulate heteronymous Renshaw cells responsible for recurrent inhibition of the quadriceps musculature. Recurrent inhibition was quantified by comparing the first peak-to-peak amplitude from the pre-synaptic protocol to the second H-reflex from the postsynaptic inhibition protocol (Bussel and Pierrot-Deseilligny, 1977, Earles et al., 2002). Eight measures were obtained, recorded, and averaged (Earles et al., 2002). 


\section{Testing Procedures}

Eligible participants reported to the General Clinical Research Center the evening prior to testing. This was done to standardise dietary (Walton et al., 2003) or diurnal variation effects (Lagerquist et al., 2006) which could influence H-reflex values. An evening meal and snack, void of caffeine or other stimulants, were provided to participants prior to going to bed no later than midnight. Participants were awoken at 5:50 AM and asked to use the restroom and then returned to their bed for 30 minutes prior to testing. This was done to provide participants with a standardised resting baseline for initial measurements. At 6:30 AM baseline H-reflex, paired reflex depression, and recurrent inhibition measurements were taken. After baseline measures were obtained participants were randomised to one of the following groups 1) lumbopelvic joint manipulation (Grade V), 2) lumbopelvic manipulation positioning (no thrust), 3) patellar mobilisation (Grade IV), 4) patellar mobilisation (Grade I), and 5) control (no intervention) using concealed allocation with assignment in sealed envelopes. The interventions were delivered by a physical therapist with advanced manual therapy training and over 20 years of clinical experience.

Investigators gathering outcomes data and investigators responsible for interpreting the outcomes data were blinded to treatment group allocation. Following intervention the investigator returned to collect post-intervention H-reflex measurements $(0,30,60,90 \mathrm{~min}$ post-intervention) using the same methods as listed above.

Lumbopelvic joint manipulation (Grade V)—The lumbopelvic joint manipulation (Figure 2) was performed on the same side as the test limb and was consistent with previous methods (Flynn et al., 2002, Grindstaff et al., 2012). If a cavitation was heard or felt by the participant or physical therapist, post-intervention testing of H-reflex parameters continued. If a cavitation was not heard or felt by the participant or physical therapist, the intervention was repeated. If the second attempt did not produce cavitation the procedures were repeated on the opposite side. A maximum of four manipulations were attempted, if cavitation was not heard or felt by the participant or physical therapist, testing of H-reflex parameters continued as usual.

Lumbopelvic manipulation positioning (no thrust)-This technique mimicked the positioning used for the lumbopelvic joint manipulation. Participants were only rotated through approximately half of range of motion and the technique was performed without the delivery of a posterior/inferior force through the ASIS. No participant experienced cavitation with this intervention. Following the positioning, participants were passively returned to the supine position and testing of H-reflex parameters continued as usual.

Patellar mobilisation (Grade IV)—The ipsilateral patella was mobilised using a technique common for individuals with knee pain (Cowan et al., 2003, Hengeveld and Banks, 2005, McConnell, 1986) (Figure 3). The mobilisation was performed by first engaging the end limit of medial patella excursion. Next, small amplitude oscillations in a medial direction were performed at the end limit of the available motion and stressed into tissue resistance. The mobilisation was performed for two minutes with approximately 1 oscillation per second. 
Patellar mobilisation (Grade I)—Patient position and physical therapist hand placement were identical to the Grade IV mobilisation (Figure 3). A grade I mobilisation was performed using small amplitude rhythmic oscillations performed at the beginning of the range of movement and did not engage the end range of motion. The mobilisation was performed for two minutes with approximately 1 oscillation per second.

Control-no intervention-The participant remained in a supine position on treatment table for three minutes and no intervention was performed by the physical therapist. Three minutes was selected to allow the physical therapist to remain in the room with the participant for a period of time similar to the other interventions. The therapist did not touch the participant in order to avoid any cutaneous stimulation, which may affect spinal reflex excitability.

\section{Statistical analysis}

The primary outcome variables were the pre-intervention to post-intervention changes in: (a) quadriceps spinal reflex excitability (Hoffmann reflex), (b) presynaptic inhibition (paired reflex depression), and (c) postsynaptic inhibition (recurrent inhibition). The data for each primary outcome variable were analysed by way of a hierarchical linear-mixed model for repeated measures.

In regard to hypothesis testing, in order to control for false positive findings (i.e. type I error) we first conducted two global Type II F-tests to determine: 1) if the total variability in the post-intervention outcome measurements explained by the "intervention" and its associated interactions was beyond what would be expected by chance, and 2) if the total variability in the post-intervention outcome measurements explained by the "measurement assessment time" and its associated interactions was beyond what would be expected by chance. If one, or both of the global Type II F-tests was/were rejected at the $\mathrm{P} \leq 0.05$ level of statistical significance, then the appropriate set of post-hoc multiple comparison tests were carried out via linear contrasts of the least-squared means, utilising a two-sided Bonferroni corrected $\mathrm{P} \leq 0.05$ null hypothesis rejection criterion. The MIX procedure of the statistical software package; SAS 9.2 version (SAS Institute Inc, Cary, NC), was used to conduct the linear-mixed model analyses.

\section{Sample Size Estimation}

Our sample size calculation showed that 75 participants (i.e. 15 participants assigned to each intervention) would be required in order to have at least 0.80 statistical power to detect a $9.0 \%$ mean pre-intervention to post intervention change in muscle inhibition (either presynaptic, or postsynaptic inhibition) under the assumption that the true standard deviation for the percentage change in muscle inhibition is no greater than previously reported values of $12.3 \%$ (Suter et al., 1999). This is a different measurement technique than we used but it represents a similar physiological process. A two-sided type I error rate of 0.05 was utilised in the sample size calculation. 


\section{RESULTS}

Participant demographics are reported in Table 1. One participant (patellar mobilizationgrade IV) was excluded from postsynaptic data analysis due to inconsistent measures at each testing interval. The specific number of lumbopelvic joint manipulation attempts to produce cavitation was not tracked since cavitation may not be necessary to achieve clinically relevant outcomes (Flynn et al., 2006, Flynn et al., 2003) or changes in quadriceps activation (Grindstaff et al., 2012).

\section{Hoffmann reflex}

The total variability in the pre-intervention to post-intervention change in quadriceps $\mathrm{H} / \mathrm{M}$ ratio that was explained by the intervention group assignment was no greater than what would be expect by chance (global F-test; $\mathrm{F}_{16,70}=0.94, \mathrm{P}=0.53$ ). Likewise, the total variability in the pre-intervention to post-intervention changes in quadriceps $\mathrm{H} / \mathrm{M}$ ratio explained by the post-intervention measurement assessment times was no greater than what would be expect by chance (global F-test; $F_{15,70}=0.76, P=0.72$, Table 2). Therefore, significant intervention versus intervention differences in the mean pre-intervention to postintervention change in quadriceps $\mathrm{H} / \mathrm{M}$ ratio was not support by our data.

\section{Paired reflex depression-presynaptic inhibition}

The total variability in the pre-intervention to post-intervention change in pre-synaptic inhibition of the quadriceps muscle that was explained by the intervention group assignment was no greater than what would be expect by chance (global F-test; $\mathrm{F}_{16,70}=0.75, \mathrm{P}=0.73$ ). Similarly, the total variability in the pre-intervention to post-intervention change in presynaptic inhibition of the quadriceps muscle that was explained by the post-intervention measurement assessment times was no greater than what would be expected by chance (global F-test; $\mathrm{F}_{15,70}=0.89, \mathrm{P}=0.58$, Table 3). Therefore, significant intervention versus intervention differences in the mean pre-intervention to post-intervention change in presynaptic inhibition was not support by our data.

\section{Recurrent inhibition-postsynaptic inhibition}

Significant variability in the pre-intervention to post-invention change in recurrent inhibition of the change quadriceps muscle was due to the intervention group assignment (global Ftest; $\mathrm{F}_{16,70}=1.87, \mathrm{P}=0.04$ ) and significant variability in the pre-intervention to post-invention change in recurrent inhibition of the change quadriceps muscle was due to the postintervention measurement assessment time (global F-test; $F_{15,70}=2.36, P=0.001$ ). However, post-hoc test examination with correction for multiple comparisons showed that the magnitudes of the intervention versus intervention differences in the mean pre-intervention to post-invention change were too small to rule out false positive findings (Table 3).

\section{DISCUSSION}

The results of this study indicate manual therapies directed at the knee or lumbopelvic region did not have an immediate effect on spinal reflex excitability of the quadriceps in individuals with knee pathology and quadriceps inhibition. This was the first study to 
directly compare changes in spinal excitability and quantify the temporal effects following manual therapy interventions directed at the knee and lumbopelvic region in a highly controlled environment. Knee joint mobilisation has been shown to decrease the flexor withdraw reflex and hamstring EMG activity (Courtney et al., 2010). Differences between our study and Courtney et al 2010 may have been due to methodological differences in estimates of spinal reflex excitability. The flexor withdraw reflex examines the contribution of the hamstring musculature following the application of a noxious stimulus to the plantar surface of the foot (Courtney et al., 2010), while this study examined the quadriceps muscle using H-reflex methodology. It is possible the changes in spinal excitability following knee injury are different between hamstring and quadriceps musculature (Torry et al., 2000). The relationship between these muscles and the duration of changes in pain-reflex excitability has not been quantified.

Changes in voluntary quadriceps activation have been shown to occur immediately following lumbopelvic manipulation (Grindstaff et al., 2009, Suter et al., 1999, Suter et al., 2000) and may last up to 20 minutes (Grindstaff et al., 2009). Applying manual therapy interventions at the lumbopelvic region has been shown to briefly decrease ( $<1$ minute) spinal excitability of the gastrocnemius and soleus, measured using H-reflex techniques (Dishman and Bulbulian, 2000, Murphy et al., 1995, Suter et al., 2005). The interventions and measures of spinal reflex excitably of the quadriceps in this study were all performed with the participant in a supine position, thus controlling for any potential changes that occur due to repositioning (Suter et al., 2005). The contribution of changes in spinal reflex excitability as a mechanism to increase voluntary quadriceps activation remains elusive.

Previous studies (Courtney et al., 2010, Moss et al., 2007) which have increased pain thresholds via joint mobilisation of the knee joint have applied the intervention for 6-9 minutes. A limitation of this study was the relatively short duration for mobilisation interventions, which may have not been of sufficient duration to influence spinal reflex excitability. It is not clear if joint mobilisation needs to be applied for greater duration to alter efferent motor excitability or if there is a relatively short window of effectiveness $(<1$ minute) (Dishman and Bulbulian, 2000, Murphy et al., 1995, Suter et al., 2005). Interventions such as cryotherapy and transcutaneous electrical stimulation have demonstrated the ability to increase quadriceps H-reflex (Hopkins et al., 2002) and voluntary quadriceps activation (Pietrosimone et al., 2009, Pietrosimone and Ingersoll, 2009, Pietrosimone et al., 2011b) when applied for 20-30 minutes. Future studies should determine if altering treatment dosing (intensity and duration) impact measured outcomes including pain thresholds, efferent motor output, and joint motion.

A limitation of this study was individuals were not currently seeking medical care for knee pain. It is possible individuals who have greater levels of pain or dysfunction may respond differently as improvements in function and quadriceps activation following manual therapy interventions directed at the spine have been demonstrated in previous studies with symptomatic participants who were seeking medical care (Iverson et al., 2008, Suter et al., 1999, Suter et al., 2000). Since this was a mechanistic study and only focused on changes in spinal excitability of the quadriceps muscle, changes in pain and function were not monitored or quantified. Another limitation of this study was we did not assess joint 
mobility (arthrokinematics) prior to or following intervention. The interventions utilized were a specific subset of possible manual therapy interventions and were applied in a randomized manner. It is possible individuals with limitations in joint mobility would derive greater benefit from specific manual therapy interventions directed at the site of dysfunction (Deyle et al., 2005) versus a generalised manual therapy approach. Future studies should perform assessment of joint arthrokinematics and consider selection of interventions to address specific impairments based on examination findings.

\section{CONCLUSIONS}

Manual therapies directed at the knee or lumbopelvic region did not have an immediate effect of quadriceps spinal reflex excitability. Although manual therapies may improve impairments and functional outcomes the underlying mechanism does not appear to be related to changes in spinal reflex excitability.

\section{Acknowledgments}

We would like to thank James R. Beazell, DPT, ATC, FAAOMPT for his manual therapy expertise and assistance with the interventions. This study was supported by grant 5R21AT004171-02 (PI: Ingersoll) from the National Center for Complementary and Alternative Medicine (NIH-NCCAM).

\section{REFERENCES}

Bussel B, Pierrot-Deseilligny E. Inhibition of human motoneurones, probably of renshaw origin, elicited by an orthodromic motor discharge. J Physiol. 1977; 269:319-339. [PubMed: 894596]

Chmielewski TL, Stackhouse S, Axe MJ, Snyder-Mackler L. A prospective analysis of incidence and severity of quadriceps inhibition in a consecutive sample of 100 patients with complete acute anterior cruciate ligament rupture. J Orthop Res. 2004; 22:925-930. [PubMed: 15304261]

Courtney CA, Lewek MD, Witte PO, Chmell SJ, Hornby TG. Heightened flexor withdrawal responses in subjects with knee osteoarthritis. J Pain. 2009; 10:1242-1249. [PubMed: 19628435]

Courtney CA, Witte PO, Chmell SJ, Hornby TG. Heightened flexor withdrawal response in individuals with knee osteoarthritis is modulated by joint compression and joint mobilization. $\mathrm{J}$ Pain. 2010; 11:179-185. [PubMed: 19945353]

Cowan SM, Bennell KL, Hodges PW, Crossley KM, McConnell J. Simultaneous feedforward recruitment of the vasti in untrained postural tasks can be restored by physical therapy. J Orthop Res. 2003; 21:553-558. [PubMed: 12706031]

Deyle GD, Allison SC, Matekel RL, Ryder MG, Stang JM, Gohdes DD, et al. Physical therapy treatment effectiveness for osteoarthritis of the knee: A randomized comparison of supervised clinical exercise and manual therapy procedures versus a home exercise program. Phys Ther. 2005; 85:1301-1317. [PubMed: 16305269]

Dishman JD, Ball KA, Burke J. Central motor excitability changes after spinal manipulation: A transcranial magnetic stimulation study. J Manipulative Physiol Ther. 2002a; 25:1-9. [PubMed: 11898013]

Dishman JD, Bulbulian R. Spinal reflex attenuation associated with spinal manipulation. Spine. 2000; 25:2519-2524. [PubMed: 11013505]

Dishman JD, Cunningham BM, Burke J. Comparison of tibial nerve h-reflex excitability after cervical and lumbar spine manipulation. J Manipulative Physiol Ther. 2002b; 25:318-325. [PubMed: 12072852]

Dishman JD, Dougherty PE, Burke JR. Evaluation of the effect of postural perturbation on motoneuronal activity following various methods of lumbar spinal manipulation. Spine J. 2005; 5:650-659. [PubMed: 16291107] 
Earles DR, Dierking JT, Robertson CT, Koceja DM. Pre- and post-synaptic control of motoneuron excitability in athletes. Med Sci Sports Exerc. 2002; 34:1766-1772. [PubMed: 12439081]

Flynn T, Fritz J, Whitman J, Wainner R, Magel J, Rendeiro D, et al. A clinical prediction rule for classifying patients with low back pain who demonstrate short-term improvement with spinal manipulation. Spine. 2002; 27:2835-2843. [PubMed: 12486357]

Flynn TW, Childs JD, Fritz JM. The audible pop from high-velocity thrust manipulation and outcome in individuals with low back pain. J Manipulative Physiol Ther. 2006; 29:40-45. [PubMed: 16396728]

Flynn TW, Fritz JM, Wainner RS, Whitman JM. The audible pop is not necessary for successful spinal high-velocity thrust manipulation in individuals with low back pain. Arch Phys Med Rehabil. 2003; 84:1057-1060. [PubMed: 12881834]

Grindstaff TL, Hertel J, Beazell JR, Magrum EM, Ingersoll CD. Effects of lumbopelvic joint manipulation on quadriceps activation and strength in healthy individuals. Man Ther. 2009; 14:415-420. [PubMed: 18805726]

Grindstaff TL, Hertel J, Beazell JR, Magrum EM, Kerrigan DC, Fan X, et al. Lumbopelvic joint manipulation and quadriceps activation of people with patellofemoral pain syndrome. J Athl Train. 2012; 47:24-31. [PubMed: 22488227]

Hengeveld, E.; Banks, K. Maitland's Peripheral Manipulation. 4th ed.. Edinburgh: Elsevier; 2005.

Hillermann B, Gomes AN, Korporaal C, Jackson D. A pilot study comparing the effects of spinal manipulative therapy with those of extra-spinal manipulative therapy on quadriceps muscle strength. J Manipulative Physiol Ther. 2006; 29:145-149. [PubMed: 16461174]

Hopkins J, Ingersoll CD, Edwards J, Klootwyk TE. Cryotherapy and transcutaneous electric neuromuscular stimulation decrease arthrogenic muscle inhibition of the vastus medialis after knee joint effusion. J Athl Train. 2002; 37:25-31. [PubMed: 12937440]

Hopkins JT, Ingersoll CD. Arthrogenic muscle inhibition: A limiting factor in joint rehabilitation. J Sport Rehabil. 2000; 9:135-159.

Hopkins JT, Ingersoll CD, Krause BA, Edwards JE, Cordova ML. Effect of knee joint effusion on quadriceps and soleus motoneuron pool excitability. Med Sci Sports Exerc. 2001; 33:123-126. [PubMed: 11194097]

Hopkins JT, Wagie NC. Intrasession and intersession reliability of the quadriceps hoffmann reflex. Electromyogr Clin Neurophysiol. 2003; 43:85-89. [PubMed: 12661132]

Ingersoll CD, Grindstaff TL, Pietrosimone BG, Hart JM. Neuromuscular consequences of anterior cruciate ligament injury. Clin Sports Med. 2008; 27:383-404. [PubMed: 18503874]

Iverson CA, Sutlive TG, Crowell MS, Morrell RL, Perkins MW, Garber MB, et al. Lumbopelvic manipulation for the treatment of patients with patellofemoral pain syndrome: Development of a clinical prediction rule. J Orthop Sports Phys Ther. 2008; 38:297-312. [PubMed: 18515959]

Kameyama O, Hayes KC, Wolfe D. Methodological considerations contributing to variability of the quadriceps h-reflex. Am J Phys Med Rehabil. 1989; 68:277-282. [PubMed: 2590515]

Lagerquist O, Zehr EP, Baldwin EL, Klakowicz P, Collins D. Diurnal changes in the amplitude of the hoffmann reflex in the human soleus but not in the flexor carpi radialis muscle. Exp Brain Res. 2006; 170:1-6. [PubMed: 16328299]

McConnell J. The management of chondromalacia patellae: A long-term solution. Aust J Physiother. 1986; 32:215-223.

McLeod MM, Gribble PA, Pfile KR, Pietrosimone BG. Effects of arthroscopic partial meniscectomy on quadriceps strength: A systematic review. J Sport Rehabil. 2012; 21:285-295. [PubMed: 22234935]

Moss P, Sluka K, Wright A. The initial effects of knee joint mobilization on osteoarthritic hyperalgesia. Man Ther. 2007; 12:109-118. [PubMed: 16777467]

Murphy BA, Dawson NJ, Slack JR. Sacroiliac joint manipulation decreases the h-reflex. Electromyogr Clin Neurophysiol. 1995; 35:87-94. [PubMed: 7781578]

Palmieri RM, Ingersoll CD. Intersession reliability of a protocol to assess reflex activation history in the vastus medialis. Int J Neurosci. 2005; 115:735-740. [PubMed: 15823938] 
Palmieri RM, Tom JA, Edwards JE, Weltman A, Saliba EN, Mistry DJ, et al. Arthrogenic muscle response induced by an experimental knee joint effusion is mediated by pre- and post-synaptic spinal mechanisms. J Electromyogr Kinesiol. 2004; 14:631-640. [PubMed: 15491837]

Palmieri RM, Weltman A, Edwards JE, Tom JA, Saliba EN, Mistry DJ, et al. Pre-synaptic modulation of quadriceps arthrogenic muscle inhibition. Knee Surg Sports Traumatol Arthrosc. 2005; 13:370 376. [PubMed: 15685462]

Pietrosimone BG, Hart JM, Saliba SA, Hertel J, Ingersoll CD. Immediate effects of transcutaneous electrical nerve stimulation and focal knee joint cooling on quadriceps activation. Med Sci Sports Exerc. 2009; 41:1175-1181. [PubMed: 19461552]

Pietrosimone BG, Hertel J, Ingersoll CD, Hart JM, Saliba SA. Voluntary quadriceps activation deficits in patients with tibiofemoral osteoarthritis: A meta-analysis. PM R. 2011a; 3:153-162. [PubMed: 21333954]

Pietrosimone BG, Ingersoll CD. Focal knee joint cooling increases the quadriceps central activation ratio. J Sports Sci. 2009; 27:873-879. [PubMed: 19449251]

Pietrosimone BG, Saliba S, Hart JM, Hertel J, Kerrigan DC, Ingersoll CD. Effects of transcutaneous electrical nerve stimulation and therapeutic exercise on quadriceps activation in people with tibiofemoral osteoarthritis. J Ortho Sports Phys Ther. 2011b; 41:4-12.

Segal NA, Glass NA, Felson DT, Hurley M, Yang M, Nevitt M, et al. Effect of quadriceps strength and proprioception on risk for knee osteoarthritis. Med Sci Sports Exerc. 2010; 42:2081-2088. [PubMed: 20351594]

Snyder-Mackler L, De Luca PF, Williams PR, Eastlack ME, Bartolozzi ARI. Reflex inhibition of the quadriceps femoris muscle after injury or reconstruction of the anterior cruciate ligament. J Bone Joint Surg Am. 1994; 76:555-560. [PubMed: 8150823]

Stackhouse SK, Stevens JE, Lee SC, Pearce KM, Snyder-Mackler L, Binder-Macleod SA. Maximum voluntary activation in nonfatigued and fatigued muscle of young and elderly individuals. Phys Ther. 2001; 81:1102-1109. [PubMed: 11319935]

Stevens JE, Mizner RL, Snyder-Mackler L. Quadriceps strength and volitional activation before and after total knee arthroplasty for osteoarthritis. J Orthop Res. 2003; 21:775-779. [PubMed: 12919862]

Stokes M, Young A. The contribution of reflex inhibition to arthrogenous muscle weakness. Clin Sci (Lond). 1984; 67:7-14. [PubMed: 6375939]

Suter E, McMorland G, Herzog W. Short-term effects of spinal manipulation on h-reflex amplitude in healthy and symptomatic subjects. J Manipulative Physiol Ther. 2005; 28:667-672. [PubMed: 16326236]

Suter E, McMorland G, Herzog W, Bray R. Decrease in quadriceps inhibition after sacroiliac joint manipulation in patients with anterior knee pain. J Manipulative Physiol Ther. 1999; 22:149-153. [PubMed: 10220713]

Suter E, McMorland G, Herzog W, Bray R. Conservative lower back treatment reduces inhibition in knee-extensor muscles: A randomized controlled trial. J Manipulative Physiol Ther. 2000; 23:7680. [PubMed: 10714531]

Torry MR, Decker MJ, Viola RW, O'Connor DD, Steadman JR. Intra-articular knee joint effusion induces quadriceps avoidance gait patterns. Clin Biomech. 2000; 15:147-159.

Trimble MH, Du P, Brunt D, Thompson FJ. Modulation of triceps surae h-reflexes as a function of the reflex activation history during standing and stepping. Brain Res. 2000; 858:274-283. [PubMed: 10708678]

Walton C, Kalmar J, Cafarelli E. Caffeine increases spinal excitability in humans. Muscle Nerve. 2003; 28:359-364. [PubMed: 12929197] 


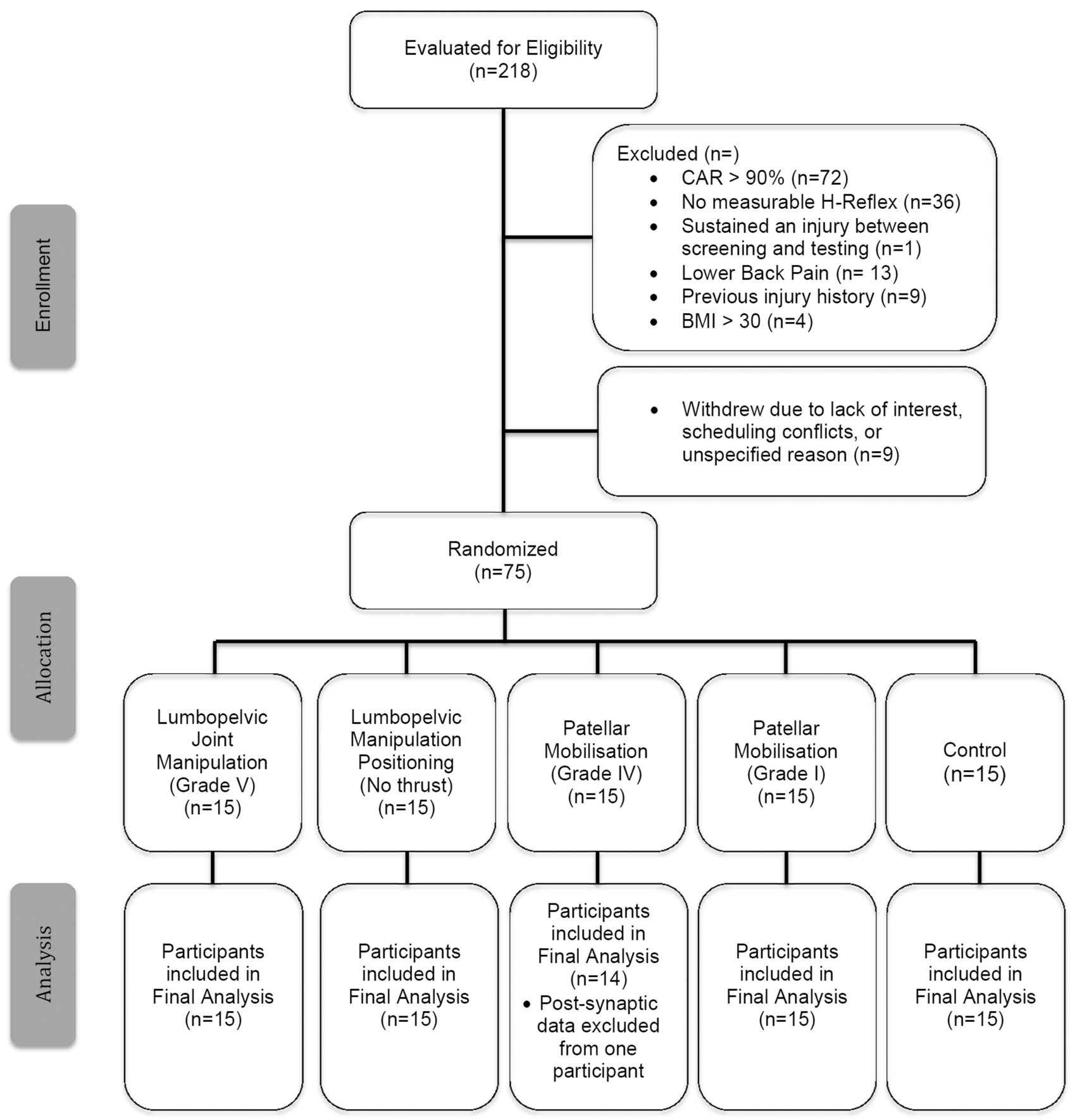

Figure 1.

CONSORT Flow Chart. 


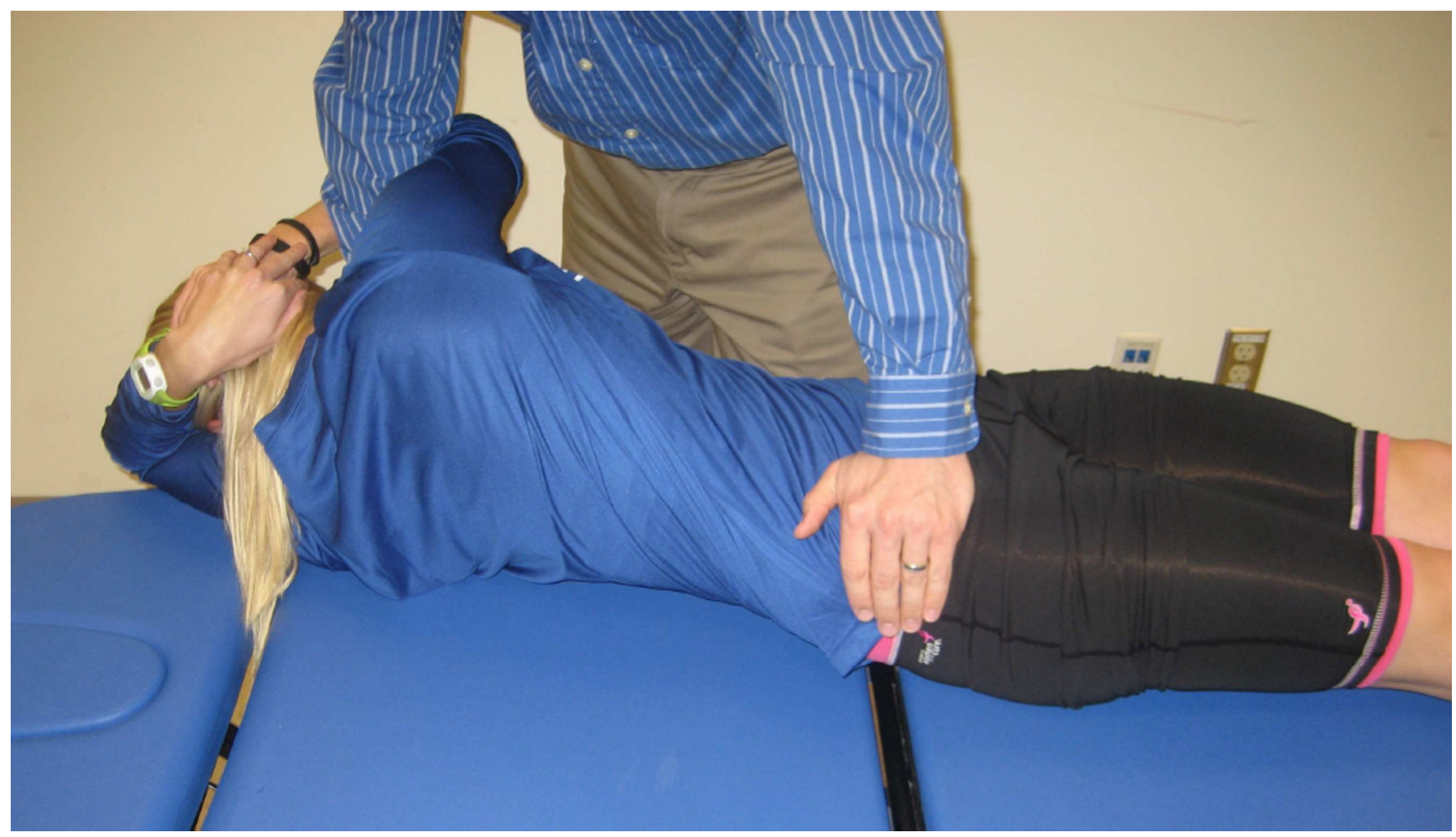

Figure 2.

Lumbopelvic joint manipulation. Participants were supine on the testing bed, while the physical therapist stood on the opposite side to be manipulated. The participant was passively side-bent away and rotated towards the physical therapist. After positioning, the physical therapist delivered a low amplitude, high velocity thrust directed through the anterior superior iliac spine (ASIS) in a posterior/inferior direction. 


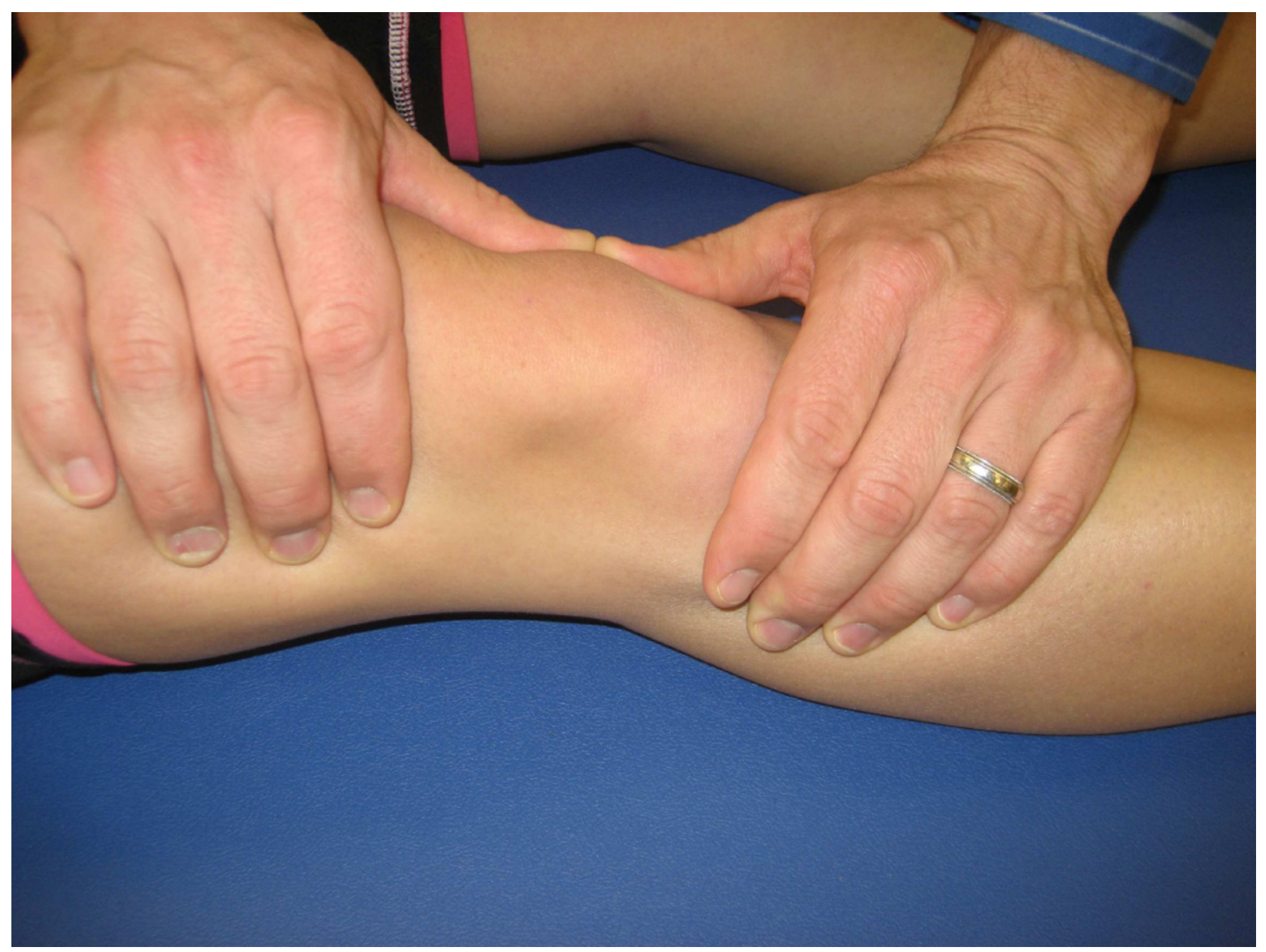

Figure 3.

Patella mobilisation (Grade IV and I). The patient was in a supine position with the knee in full extension. The physical therapist placed both thumbs on the lateral aspect of the patella while the fingers of both hands rested across the tibia and femur pointing in a medial direction. The grade IV mobilisation was performed by first engaging the end limit of medial patella excursion. Next, small amplitude oscillations in a medial direction were performed at the end limit of the available motion and stressed into tissue resistance. A grade I mobilisation was performed using small amplitude rhythmic oscillations performed at the beginning of the range of movement and did not engage the end range of motion. 


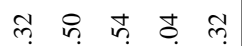

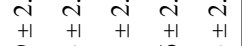

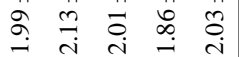

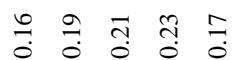
$\begin{array}{lllll}0 & 0 & 0 & 0 & 0 \\ 0 & 0 & 0 & 0 \\ +1 & +1 & +1 & +1 & +1 \\ 0 & & & & 0\end{array}$ กู่ สิ สิ สู 
